\title{
ENTRE LA IMPOSICIÓN Y LA NECESIDAD: IMPLEMENTACIÓN DEL PLAN DE FORMACIÓN CIUDADANA EN ESCUELAS CHILENAS ${ }^{1}$
}

\author{
Carmen Gloria Zúñiga ${ }^{2}$, Patricia Ojeda ${ }^{3}$, Paula Neira ${ }^{4}$, \\ Teresa Cortés ${ }^{5}$, María Jesús Morel ${ }^{6}$
}

\begin{abstract}
RESUMEN
Los resultados del estudio a los jóvenes chilenos participantes del Estudio Internacional de Educación Cívica y Ciudadanía 2016 enfatizan la necesidad de que la escuela influya positivamente en la formación de ciudadanos, lo que ha sido reforzado por la Ley $\mathrm{N}^{\circ}$ 20.911. Mediante una metodología cualitativa y siguiendo un enfoque interpretativo de estudio de caso múltiple, este artículo busca caracterizar formas de concreción de la política educativa de formación ciudadana en ocho establecimientos educacionales del país, analizando las perspectivas de directivos, profesores y estudiantes de octavo básico, prácticas de aula y el contenido de los planes de formación ciudadana. Los resultados muestran que la elaboración del Plan de Formación Ciudadana ha carecido de acompañamientos y su responsabilidad recae principalmente en el docente encargado de cada escuela, lo cual implica que, en muchos casos, no exista una reflexión colaborativa sobre el tipo de ciudadano que se quiere formar. Asimismo, se asigna la labor de formar ciudadanos a la asignatura de Historia, Geografía y Ciencias Sociales, contradiciendo el carácter transversal de la formación ciudadana en el currículo escolar. Finalmente, el Plan de Formación Ciudadana se elabora principalmente para cumplir con la normativa ministerial vigente.
\end{abstract}

Conceptos clave: formación ciudadana, política pública, prácticas de aula, percepciones docentes, percepciones estudiantes.

1 Proyecto apoyado financieramente por CNED/ Convocatoria 2018.

FONDECYT Regular No 1180667: Sistema escolar chileno y el desarrollo de resultados cívicos. Formas de implementación, mecanismos de recontextualización de la política educativa e influencia de la escuela en el conocimiento, actitudes y participación cívica de los jóvenes.

2 Pontificia Universidad Católica de Chile, Santiago, Chile. Contacto: carmen.zuniga@uc.cl

3 Pontificia Universidad Católica de Chile, Santiago, Chile. Contacto: piojeda@uc.cl

4 Pontificia Universidad Católica de Chile, Santiago, Chile. Contacto: pjneira@uc.cl

5 Pontificia Universidad Católica de Chile, Santiago, Chile. Contacto: mtcorte2@uc.cl

6 Pontificia Universidad Católica de Chile, Santiago, Chile. Contacto: mjmorel@uc.cl 


\title{
BETWEEN IMPOSITION AND NEED: IMPLEMENTATION OF THE PLAN FOR CITIZENSHIP EDUCATION IN CHILEAN SCHOOLS
}

\begin{abstract}
The results of the young Chileans who participated in the International Study of Civic and Citizenship Education 2016 emphasize the need for the school to positively influence the formation of citizens, which has been reinforced by Law No. 20.911. Following a qualitative methodology and an interpretive approach of a multiple case study, this project seeks to characterize the materialization of the educational policy of citizenship education in eight schools throughout the country. This was done based on an analysis of the Plan for Citizenship Education, observation of teacher practices, review of documents and the perception of teachers, principals and 8th grade students, gleamed from interviews. Results show that the elaboration of the Plan for Citizenship Education has lacked proper support and its responsibility lies mainly with the teacher assigned to oversee its implementation, in each school, which implies that in many cases there is no collaborative reflection on the type of citizen that should be formed. Likewise, the work of educating citizens is assigned to the subject of History, Geography and Social Sciences, contradicting the transversal nature of Citizenship Education in the school curriculum. Finally, the Citizenship Education Plan is drawn up primarily to comply with current ministerial regulations.
\end{abstract}

Key concepts: citizenship education, public policy, classroom practices, teacher perceptions, student perceptions 


\section{Introducción}

Los resultados del Estudio Internacional de Educación Cívica y Ciudadanía (ICCS) indicaron que el conocimiento cívico de los jóvenes chilenos se encuentra por debajo de la media internacional, y que entre 2009 y 2016 su conocimiento cívico disminuyó. Dichos resultados enfatizan la necesidad de que la escuela influya positivamente en la formación de ciudadanos, lo que ha sido reforzado por la Ley No 20.911. Al respecto, la investigación académica indica que los establecimientos escolares necesitan apoyo para cumplir exitosamente con la política pública de formación ciudadana, respaldo que debe estar cimentado en investigaciones contextualizadas provenientes de datos de escuelas chilenas.

Este artículo se enmarca en un proyecto de investigación, que busca caracterizar formas de concreción de la política educativa de formación ciudadana en establecimientos educacionales del país, a partir de la visión conjunta del Plan de Formación Ciudadana (PFC), el análisis de las prácticas de aula que promuevan la formación ciudadana y el levantamiento de las percepciones de directivos, docentes y estudiantes respecto del tópico. Se espera que estos hallazgos, siguiendo un enfoque interpretativo y una metodología de estudio de caso múltiple, generen conocimiento contextualizado acerca de las formas de implementación de la política pública sobre formación ciudadana, como una manera de guiar mejoras en su planificación, implementación y evaluación.

\section{Contexto y antecedentes del estudio}

La escuela representa la primera oportunidad en que un individuo puede vincularse con grupos más amplios que la familia y los vecinos. Es una instancia de aprendizaje para relacionarse con el otro sobre la base del respeto y la tolerancia, donde se promueve el desarrollo sustentable y se incentiva la participación en diferentes ámbitos. Sin embargo, la escuela no es necesariamente democrática y puede no enseñar a participar activamente en sociedad (Engel, 2015), a pesar de los numerosos intentos desde la política pública para que así sea, esfuerzos que se han traducido en programas de estudios, 
138 ENTRE LA IMPOSICIÓN Y LA NECESIDAD: IMPLEMENTACIÓN DEL PLAN DE FORMACIÓN CIUDADANA EN ESCUELAS CHILENAS - C. G. Zúñiga, P. Ojeda, P. Neira, T. Cortés, M. J. Morel

bases curriculares y planes escolares, entre otros. Lo anterior se puede deber a que los diseños curriculares implementados en Chile siguen un enfoque top-down (Olson, 2002), en el que docentes y directivos de escuelas son los responsables de dar forma a políticas educativas que les son mandatadas (Walshaw \& Anthony, 2007).

\section{La política pública de formación ciudadana en Chile}

Se han realizado numerosos esfuerzos desde el Ministerio de Educación (MINEDUC) para mejorar los procesos de formación ciudadana en las últimas dos décadas. En 1998 se comenzó a implementar un nuevo currículo que dejó atrás una visión minimalista de ciudadanía, centrada en la institucionalidad del Estado y la identidad nacional. De ser Educación Cívica una asignatura obligatoria para tercer año medio, pasó a ser un Objetivo Fundamental Transversal (OFT) para todas las asignaturas y en todos los niveles, basado en el enfoque de educación ciudadana que considera que los estudiantes forman parte de la sociedad y, por tanto, deben participar dentro del contexto en el que están inmersos (MINEDUC, 1999).

El progresivo declive en los niveles de participación política de los jóvenes condujo al MINEDUC a convocar una comisión en 2004, cuyo objetivo fue promover procesos de mejora en la formación ciudadana en el nivel escolar. Los resultados de dicha comisión se tradujeron en sugerencias para promover el área en el Ajuste Curricular 2009 (MINEDUC, 2009). A su vez, las bases curriculares de 2012 y 2013 incluyeron la formación ciudadana como un énfasis de las propuestas curriculares de la asignatura Historia, Geografía y Ciencias Sociales (en adelante HGCS), desde $1^{\circ}$ básico hasta $2^{\circ}$ medio (MINEDUC, 2013; 2015). Adicionalmente, en 2014 la Agencia de la Calidad de Educación incluyó, en los Indicadores de Desarrollo Personal y Social del SIMCE, la dimensión "Participación y Formación Ciudadana". Sin embargo, el esfuerzo más contundente se produjo en 2016, con la aprobación de la Ley No 20.911 que mandata la implementación de un Plan de Formación Ciudadana (en adelante PFC) en todos los establecimientos educacionales a partir de 2017, y la creación de una asignatura escolar en $3^{\circ}$ y $4^{\circ}$ medio desde 2020. Finalmente, el último avance en la materia ha sido la Evaluación 
Nacional de Educación Cívica y Formación Ciudadana, dirigida por la Agencia de la Calidad de Educación.

El primer objetivo de la Ley 20.911 propone la comprensión y el análisis del concepto de "ciudadanía", sin embargo, éste no se encuentra definido, más bien, se asocia al ejercicio y cumplimiento de los derechos y deberes. Con base en lo establecido por la ley, la propuesta actual del MINEDUC concibe una ciudadanía activa, relacionada con una participación enfocada en la responsabilidad y el cumplimiento de derechos y deberes, como la promoción de los derechos humanos, la protección del medioambiente y el perfeccionamiento de la democracia. Además, la relaciona con actitudes como la tolerancia, la autonomía, la solidaridad, y con habilidades como el pensamiento crítico (MINEDUC, 2016a, b).

La Ley 20.911 indica que los centros escolares deben implementar un PFC que se incorpore a todos los niveles escolares y que se conciba colaborativamente, haciendo partícipes a todos los actores del sistema escolar. El Plan enfatiza la formación ciudadana, superando el enfoque tradicional de educación cívica (MINEDUC, 2016a). Se espera que el contenido del PFC sea coherente con los principios señalados por la literatura internacional para el desarrollo de la formación ciudadana, teniendo entre sus metas el conocimiento de la sociedad, la política y la institucionalidad; el desarrollo de una ciudadanía activa, crítica y responsable; el respeto de la diversidad social y los derechos humanos, y el fomento de la participación y de una cultura de transparencia y probidad (MINEDUC, 2016a). La novedad del PFC se encuentra en las herramientas con las que se espera visibilizar la formación ciudadana en las aulas y centros escolares. Estos instrumentos consideran la realización de talleres y actividades extraprogramáticas, la apertura de los centros a la comunidad y el fomento de su democratización (MINEDUC, 2016b).

El PNUD (2018) realizó el primer estudio para conocer la puesta en marcha del Plan en las escuelas. Los resultados indicaron que, para fines de 2017, alrededor de un 30\% de los colegios no lo había implementado. De los que sí lo implementaron, en muchos casos no siguieron una dinámica de creación participativa e inclusiva. 
140 ENTRE LA IMPOSICIÓN Y LA NECESIDAD: IMPLEMENTACIÓN DEL PLAN DE FORMACIÓN CIUDADANA EN ESCUELAS CHILENAS - C. G. Zúñiga, P. Ojeda, P. Neira, T. Cortés, M. J. Morel

Los que sí lograron esta tarea han privilegiado la implementación de actividades extraprogramáticas. En definitiva, no se cuenta con gran cantidad de evidencia acerca de cómo la gestión escolar y los procesos de enseñanza son participativos y democráticos.

\section{Marco teórico conceptual}

\section{Formación ciudadana}

Tradicionalmente, la educación cívica se enfoca en la transmisión de conocimientos relacionados con la institucionalidad política, el derecho a voto y el respeto por las instituciones democráticas. Se trata de una mirada en la que los individuos deben aprender y adaptarse a nociones estáticas de la sociedad. Desde hace algunas décadas, esta perspectiva ha sido abandonada por nociones más complejas y multidimensionales de la ciudadanía y lo cívico, generando lo que actualmente se ha denominado "formación ciudadana". Este enfoque busca fomentar el compromiso de los estudiantes para aprender a vivir democráticamente y tomar conciencia de su rol como ciudadanos, desarrollando una participación activa en la sociedad. Su acento está puesto en la dimensión práctica sobre los modos en que un ciudadano toma conciencia de sus responsabilidades, busca formar ciudadanos cívicamente activos y promueve el desarrollo de las condiciones y espacios estructurales y culturales para lograr la participación (Folgueiras, Massot y Sabariego, 2008).

La formación ciudadana promueve que los estudiantes sean capaces de formar sus propias ideas y de expresar sus opiniones, entendiendo que existen múltiples perspectivas sobre temas morales y sociales. De esta manera, se aprende a lidiar con la diversidad y vivir en tolerancia (Alivernini \& Manganelli, 2011; McAvoy \& Hess, 2013; Geboers, Geijsel, Admiraal \& ten Dam, 2013).

Este enfoque no está ligado solo al dominio político de la ciudadanía, sino que también tiene un rol social vinculado a los voluntariados y a la confianza en que los ciudadanos pueden hacer una diferencia en su entorno social y protestar contra las injusticias (Haste, 2004; Torney-Purta, 2004). Asimismo, la 
formación ciudadana está comprometida a explorar y valorar las acciones para erradicar las desigualdades, la explotación, la violencia y la marginación (Ross y Vinson, 2012), entendiendo la democracia como una realidad inacabada, en constante construcción y perfeccionamiento, que contempla todos los ámbitos de la vida en sociedad y de la cotidianeidad. La formación ciudadana busca también que los estudiantes sean capaces de proponer nuevas formas de hacer frente a los diversos problemas científicos, sociales y morales (Brint, 2006).

Diversas investigaciones indican que las características estructurales de las escuelas, como el tipo de administración y la composición socioeconómica, son predictores importantes de las actitudes cívicas (Schulz, Ainley, Fraillon, Kerr \& Losito, 2010). De este modo, la formación ciudadana busca que la escuela sea un lugar donde los estudiantes vivan experiencias democráticas y se fomente la cohesión social, considerando la diversidad de orígenes y la existencia de distintos colectivos que pueden construir un proyecto cohesionado que les permita identificarse como una comunidad desde la interculturalidad (Folgueiras et al., 2008). Por medio de la participación en situaciones cotidianas, los estudiantes pueden desarrollar una imagen de sí mismos como ciudadanos reflexivos y críticos, lo que puede mejorar su participación en la sociedad (Geboers et al., 2013).

Esta propuesta de aprendizaje basada en la formación de ciudadanos implica formar a los estudiantes considerando un tipo de ciudadano ideal. Westheimer y Kahne (2004), al estudiar los programas de estudio estadounidenses, identifican diferentes tipos de ciudadanos promovidos desde la escuela: un ciudadano personalmente responsable, uno participativo y un tercero orientado a la justicia social. De esta manera, destacan que existen múltiples formas de entender qué es un buen ciudadano, lo que implica que también es múltiple la forma de concebir una buena sociedad. Educar a un ciudadano personalmente responsable implica fomentar la compasión y el compromiso en servicios voluntarios. Un ciudadano participativo participa en asuntos cívicos de la comunidad local o nacional, y se esfuerza en organizar trabajos de manera comunitaria 
142 ENTRE LA IMPOSICIÓN Y LA NECESIDAD: IMPLEMENTACIÓN DEL PLAN DE FORMACIÓN CIUDADANA EN ESCUELAS CHILENAS - C. G. Zúñiga, P. Ojeda, P. Neira, T. Cortés, M. J. Morel

y asumir compromisos colectivos. Formar un ciudadano orientado a la justicia social implica enseñar a los estudiantes a analizar cuán efectivas son las políticas sociales y económicas, fomentando sus habilidades comunicativas y la tolerancia de mantener discusiones que contengan diferentes perspectivas. A su vez, este tipo de ciudadano comparte el énfasis que se da al trabajo colectivo del ciudadano participativo, pero critica la estructura política social que genera injusticias (Westheimer \& Kahne, 2004).

Percepciones de estudiantes y docentes sobre la formación ciudadana

La evidencia ha señalado que los jóvenes de Chile se muestran desconfiados hacia la institucionalidad política y las formas tradicionales de participación, como el sufragio, pues no las perciben como una instancia vinculante, lo que se traduce en una baja participación electoral (Henríquez y Mardones, 2016). Sin embargo, también valoran la democracia y las distintas formas de participación social y comunitaria, motivados por las fallas que han ido detectando en el sistema político. Si bien se muestran menos interesados por la política, evidencian mayor participación directa o comunitaria, con la motivación de reivindicar el poder y la voz de los ciudadanos víctimas de la inequidad, la injusticia social y la falta de legitimación de derechos (Martínez, Silva y Hernández, 2010). De esta manera, la percepción estudiantil sobre formación ciudadana parece estar más cercana a un enfoque centrado en el fomento del compromiso social y la toma de conciencia del rol ciudadano que en un enfoque fundado en la institucionalidad política, ya que los espacios donde los jóvenes chilenos normalmente exhiben altos niveles de participación son aquellos asociados a actividades culturales, clubes deportivos, grupos artísticos y organizaciones voluntarias (Treviño, Villalobos, Béjares \& Naranjo, 2019).

Los resultados del Estudio Internacional de Ciudadanía de 2016, ICCS (Agencia de la Calidad de la Educación, 2018) arrojaron que los jóvenes chilenos tienen una actitud positiva frente a la igualdad de derechos y de género, revelando su interés y preocupación por temas ciudadanos. Otros estudios apuntan a que su percepción de 
"formación ciudadana" se relaciona con la responsabilidad de los ciudadanos de mantenerse informados para deliberar y actuar sobre su realidad (Martínez et al., 2010).

Respecto de la percepción docente sobre formación ciudadana, es posible evidenciar una falta de coherencia entre las intenciones declaradas por los docentes y su práctica (Cox y Castillo, 2015). Aun cuando, en su discurso, los docentes manejan una actualización sobre las finalidades ciudadanas de la enseñanza, las estrategias utilizadas en el aula no dan espacio a interacciones democráticas y son más bien cercanas a modelos tradicionales y conductistas de enseñanza (Cavieres y Muñoz, 2015). En la práctica existe un enfoque tradicional a la hora de enseñar, en el que se entiende al docente como el centro de la clase, otorgando limitadas oportunidades de participación estudiantil en la construcción de conocimiento (Bonhomme, Cox, Tham y Lira, 2015).

\section{Prácticas docentes para la formación de ciudadanos}

Existe consenso al indicar que la formación inicial docente y las investigaciones sobre enseñanza han priorizado ahondar en aquello que los profesores saben y piensan, en vez de indagar en el conocimiento acerca de cómo enseñar aquello que saben, tendencia que Shulman (1986) identificó hace más de treinta años.

A su vez, existe uniformidad al indicar que la evaluación de la calidad de la enseñanza es un proceso sumamente complejo, pues depende del contexto en que ésta se realiza, ya que los procesos de enseñanza y aprendizaje se circunscriben a un ambiente específico. Es decir, independiente de los elementos que se conjuguen, la calidad de la enseñanza está fuertemente ligada al contexto local y cultural en que se desarrolla, por lo que cualquier clasificación debe incluir y examinar estos aspectos (Martinez, Taut $\&$ Schaaf, 2016). De este modo, no es posible encasillar prácticas sobresalientes mediante una definición única y global que sirva a todos los docentes por igual. Es más, un profesor puede presentar prácticas altamente efectivas en el desarrollo de una unidad pedagógica y en otras no mostrarse tan efectivo (Darling-Hammond, Amrein-Beardsley, Haertel \& 
144 ENTRE LA IMPOSICIÓN Y LA NECESIDAD: IMPLEMENTACIÓN DEL PLAN DE FORMACIÓN CIUDADANA EN ESCUELAS CHILENAS - C. G. Zúñiga, P. Ojeda, P. Neira, T. Cortés, M. J. Morel

Rothstein, 2012). En este sentido, más que crear una definición única de buenas prácticas para la formación de ciudadanos, este estudio busca levantar elementos que las constituyen, entendiendo que ellas no se manifiestan en todos los docentes por igual.

Ante la preponderancia del modelo de ciudadanía que requiere del dominio no solo de conocimientos, sino también de habilidades, destrezas, actitudes y comportamientos, se impone necesariamente un cambio en el modelo tradicional de enseñanza y aprendizaje. En este sentido, aunque se cuenta con un importante bagaje teórico sobre educación ciudadana, fundamentalmente a través de investigaciones sobre concepciones y currículos, son aún pocas las investigaciones sobre su aplicación pedagógica (Hahn, 2016; Zúñiga, Cárdenas, Martínez \& Valledor, 2020). En general, se advierte que la enseñanza y aprendizaje de la ciudadanía va más allá de la transmisión y adquisición de contenidos, pues debe centrarse en una práctica activa (Puig y Morales, 2015), en la que los estudiantes puedan discutir y demostrar sus aprendizajes de forma democrática en el aula. La clave es generar un aula abierta y participativa (Knowles $\&$ McCafferty-Wright, 2015; Avery, Levy \& Simmons, 2013), con aprendizajes experimentales, colaborativos, interculturales, activos y contextualizados (Keating-Chetwynd, 2009).

De esta forma, los estudiantes deben acostumbrarse a la realización de actividades participativas — como asambleas- y a escuchar, examinar, debatir y respetar puntos de vista, promover la toma de decisiones en conjunto y por el bien común, e investigar sobre nuevos datos que aporten soluciones a problemáticas sociales (Hahn, 2016). Además, ante el peso que en las democracias actuales empiezan a tener las plataformas y redes sociales, la alfabetización digital y el uso de las nuevas tecnologías deben tener un papel principal en la educación ciudadana (Haste, Bermudez \& Carretero, 2017; Kahne, Hodgin \& Eidman-Aadahl, 2016).

Por lo tanto, se requiere de un docente actualizado, que sea promotor de dinámicas en el aula, generando interacción, participación y reflexión a través de actividades variadas (Hahn, 2016; Muñoz, Martínez y Muñoz, 2016). En la formación ciudadana, el 
profesor ha de asumir un papel de guía, facilitando que sean los estudiantes los protagonistas mediante la planificación de actividades de aprendizaje activo, proponiendo proyectos ligados al contexto, fomentando procesos de reflexión y análisis, confiando en las capacidades de los estudiantes y controlando que los procesos de deliberación sean democráticos (Cárdenas, 2017). Además, el manejo de un adecuado clima en el aula, abierto a la discusión y participativo, se configura como otro elemento clave de una formación ciudadana efectiva (Hahn, 2016; Hess \& McAvoy, 2015; Schulz et al., 2010).

Asimismo, los centros educativos también son una pieza fundamental de este proceso formativo. Deben promover espacios para la experiencia social y la práctica ciudadana, fomentando la democracia, generando programas que conviertan la escuela en un lugar ligado a la comunidad y promotor de aprendizajes reales y contextualizados (Abs, 2009; Luengo \& Jiménez, 2017).

\section{Metodología}

La investigación propuesta utilizó una metodología cualitativa, ya que centró su atención en caracterizar formas de implementación de la política pública sobre formación ciudadana en establecimientos educacionales con características disímiles entre sí. Para ello, usó un enfoque interpretativo y de estudio de caso múltiple, cuyo objetivo persiguió revelar los diferentes significados construidos por las personas en un contexto social determinado (Gubrium \& Holstein, 2005), generando explicaciones aplicables a todos los casos, trascendiendo las características particulares (Merriam, 2009).

\section{Muestra y participantes}

Se seleccionó a ocho escuelas, utilizando un muestreo de variación máxima (Litchman, 2010) a partir de la composición socioeconómica y étnica, dependencia administrativa, tamaño y región de procedencia de los establecimientos, con la intención de identificar casos interesantes para la investigación, en los que fuera posible caracterizar las prácticas de formación ciudadana y las percepciones sobre ciudadanía de los distintos actores en contextos diversos. 
La tabla 1 resume las características de las escuelas participantes. Las escuelas seleccionadas presentan distintas características; a modo general, más de la mitad corresponde a establecimientos de la Región Metropolitana (RM), y el resto a las regiones de Los Lagos, La Araucanía y Valparaíso. Los establecimientos son de distinta dependencia administrativas: municipales, particulares subvencionados y particulares pagados. El tamaño de las escuelas también es disímil: la más pequeña presenta una matrícula de 137 alumnos y la más grande de alrededor de 1.600 estudiantes. A su vez, una escuela es solo de niñas y en otra la mayoría de sus estudiantes son de origen mapuche.

Tabla 1.

Características de escuelas participantes.

\begin{tabular}{clllc}
\hline N ${ }^{\circ}$ Escuela & \multicolumn{1}{c}{ Región } & \multicolumn{1}{c}{ Comuna } & \multicolumn{1}{c}{ Dependencia } & Matrícula Total \\
\hline 1 & RM & La Florida & Municipal & 566 \\
\hline 2 & Los Ríos & Valdivia & Municipal & 856 \\
\hline 3 & Valparaíso & San Antonio & Municipal & 597 \\
\hline 4 & RM & Providencia & Particular pagada & 1.599 \\
\hline 5 & RM & Talagante & Municipal & 947 \\
\hline 6 & Araucanía & Lautaro & Part. subvencionada & 135 \\
\hline 7 & RM & San Ramón & Part. subvencionada & 483 \\
\hline 8 & RM & San Joaquín & Part. subvencionada & 539 \\
\hline
\end{tabular}

Fuente: elaboración propia.

Se seleccionó a las escuelas participantes a partir de información de las bases de datos utilizadas en estudios realizados en la Facultad de Educación de la Pontificia Universidad Católica de Chile, lo que facilitó el acceso y la disposición de los establecimientos a participar. Como complemento, se consultó las siguientes fuentes de información: Directorio Oficial de Establecimientos Educacionales 2018; Resumen de Matrícula por establecimiento 2018; Resumen de Alumnos Prioritarios y Beneficiarios SEP por establecimiento 2017; Registro matrícula única 2017 por estudiante, además de otros datos disponibles en el portal Más Información Mejor Información (MIME) del MINEDUC y en los proyectos educativos institucionales de cada establecimiento, obtenidos mediante los sitios web. 
En cada escuela se seleccionó a un directivo, al encargado del PFC y a cuatro docentes, correspondientes a Orientación, Lenguaje, Comunicación y Literatura, Ciencias Naturales e HGCS. Esta selección se justifica porque en Orientación e HGCS el contenido se vincula principalmente con temáticas de formación ciudadana, ya sea en términos de conocimiento, como en actitudes y participación; además, Orientación o Consejo de Curso son asignaturas en las que los estudiantes se organizan en términos de participación y de transmisión de valores. Respecto de Lenguaje, Comunicación y Literatura, y Ciencias Naturales, esta elección se realizó sobre la base de criterios pedagógicos, dadas las metodologías que pudieran estar usando los docentes (discusión abierta en la sala de clases, participación, entre otras).

Además de los docentes, se seleccionó a un grupo de estudiantes de $8^{\circ}$ básico ${ }^{7}$. Las razones se centran en el supuesto de que los estudiantes ya han tenido algún tipo formación ciudadana, ya sea a través de clases específicas de HGCS o de manera transversal en su proceso de formación escolar. Además, hemos seguido como ejemplo el Estudio Internacional de Ciudadanía (ICCS), que trabaja con una muestra de estudiantes de $8^{\circ}$ básico.

\section{Procedimiento de recolección y análisis de datos}

Cada escuela fue considerada un caso en el que se desarrollaron tres acercamientos analíticos. Primero, se realizó un análisis acerca de cómo se promueve la formación ciudadana, mediante la observación de aula en los cursos de $8^{\circ}$ año básico en las asignaturas de Lenguaje, Comunicación y Literatura; HGCS; Ciencias Naturales y Orientación. Se analizaron cuatro horas pedagógicas por asignatura en cada establecimiento, a excepción de la asignatura de Orientación, la cual fue observada solo dos horas pedagógicas, debido a que cuenta con menos disposición horaria en la asignación curricular. El foco del análisis se centró en los procesos de promoción de la formación

7 Los participantes no serán diferenciados por género, por lo que las palabras en plural (las escuelas, los docentes, los estudiantes, etc.) incluyen a mujeres y hombres por igual. Además, los resultados no muestran diferencias ni desigualdades entre hombres y mujeres, por lo que no se incorpora el enfoque de género en el análisis. 
ciudadana en las clases, ahondando particularmente en la aplicación de metodologías de aprendizaje activo y en cómo se enseña a discutir puntos de vista distintos. Para ello se grabaron las clases y se estableció una pauta de observación para su análisis, la cual contempla siete dimensiones que se indican la tabla 2.

Tabla 2.

Dimensiones pauta de observación de clases.

\begin{tabular}{l} 
(1) Promoción intencionada de la formación ciudadana dentro del aula por el/la \\
profesor/a de historia \\
\hline (2) \\
Promoción intencionada de la formación ciudadana dentro del aula de todos \\
los profesores
\end{tabular}

Fuente: elaboración propia.

Posteriormente, se analizó la recontextualización de la política pública de formación ciudadana en la escuela. Para ello, se llevó a cabo una revisión documental de los PFC presentados por las escuelas, mediante la codificación y categorización de los principales conceptos relativos a "ciudadanía" presentes en los planes. Esto tenía por objetivo develar las relaciones conceptuales puestas en juego en la elaboración de estos programas. Para ello se utilizó una Pauta de Análisis de Documentos, que sintetizó las ideas generales del PFC y analizó la coherencia entre los objetivos y las descripciones de las acciones propuestas. Además, en las entrevistas realizadas a docentes y directivos se preguntó acerca del PFC, para complementar el discurso de los planes descritos con el de los encargados de su implementación.

Finalmente, se analizaron las formas de desarrollo de la formación ciudadana, considerando el vínculo de la escuela con la comunidad, la participación de los diferentes actores de la comunidad educativa, formas de desarrollo de la democracia escolar, entre otros aspectos. Para pesquisar esta información, se llevaron a cabo entrevistas semiestructuradas con actores de la comunidad escolar 
y se establecieron nueve dimensiones que configuraron las distintas pautas de entrevistas, presentes en la tabla 3.

Tabla 3.

Dimensiones de los instrumentos de recolección de información.

\begin{tabular}{ll}
\hline \multicolumn{1}{c}{ Dimensión } & \multicolumn{1}{c}{ Instrumento } \\
\hline Relaciones entre los distintos actores de la escuela & $\begin{array}{l}\text { Entrevista a director } \\
\text { Entrevista a encargado del PFC } \\
\text { Entrevistas a docentes } \\
\text { Entrevista grupal a estudiantes }\end{array}$ \\
\hline Relación entre escuela y comunidad & Entrevista a director \\
\hline Contexto escolar & Entrevista a encargado del PFC \\
\hline Política pública de formación ciudadana & Entrevista a director \\
\hline Promoción intencionada de la formación ciudadana & Entrevista a encargado del PFC \\
fuera del aula & Entrevista a director \\
\hline Promoción intencionada a docentes la formación ciudadana & Entrevistas a docentes \\
dentro del aula & \\
\hline Vida ciudadana fuera de la escuela & Entrevista grupal a estudiantes \\
\hline Prácticas ciudadanas dentro de la escuela & Entrevista grupal a estudiantes \\
\hline & Entrevista a director \\
Concepciones de ciudadanía & Entrevista a encargado del PFC \\
& Entrevistas a docentes \\
\hline
\end{tabular}

\section{Procedimiento de análisis de datos}

Siguiendo los procedimientos de análisis de estudios de casos múltiples, primero se analizó como un caso cada escuela de forma individual. Al examinar los datos, se utilizaron métodos de análisis de información consistentes con el enfoque interpretativo. Para cada set de datos, los procesos de recolección y análisis de información fueron llevados a cabo de manera independiente y simultánea (Charmaz, 2006; Corbin \& Strauss, 2008). Esto permitió focalizar la atención del análisis en el surgimiento de conceptos, así como en la validación de tales conceptos a medida que surgían entre los datos entregados por nuevas fuentes de información (Merriam, 2009).

Se comenzó por recolectar la información, transcribirla, clasificarla y categorizarla (Flick, 2009). En este proceso, los datos 
fueron leídos detenida y profundamente. El equipo de investigación registró comentarios, observaciones y preguntas en un diario de investigación con el formato de bitácora (Corbin \& Strauss, 2008). A medida que se recopiló más información, ésta fue constantemente comparada en cuanto a similitudes y diferencias. De este modo, surgieron apreciaciones al comparar y cuestionar los datos de manera constante (Merriam, 2009). Para ello, se realizaron reuniones grupales en las que todos los investigadores pudieron compartir y comparar sus apreciaciones; este proceso apuntó a la elaboración de conceptos y proposiciones que se ajustaran a la información obtenida, y las conjeturas hechas por los investigadores fueron exploradas, analizadas y cuestionadas constantemente (Corbin \& Strauss, 2008). Posteriormente, los conceptos y proposiciones generadas fueron clasificados en categorías, para luego describir e integrar la información en un todo coherente. Códigos, notas y diagramas fueron utilizados por el equipo de investigación para ilustrar la relación entre conceptos y categorías. Luego, la información, organizada en conceptos y categorías, fue analizada más a fondo, utilizando la forma de análisis denominada "inducción analítica", que implica la formulación de propuestas de manera sistemática (Hammersley \& Atkinson, 1995).

Finalizado el análisis de cada establecimiento, se procedió a elaborar explicaciones generales que "calzaran" para cada uno de los casos (Merriam, 2009). Para este análisis multicaso, se estudiaron procesos y resultados que pueden haber ocurrido en más de un caso, comprendiendo cómo afectan sus características contextuales $y$, por lo tanto, elaborando descripciones más sofisticadas que permitieron descubrir patrones y explicaciones que trascienden los casos individuales (Merriam, 2009).

\section{Resultados}

El proceso de análisis por caso y multicaso permitió establecer categorías generales que pueden aplicarse transversalmente a las ocho escuelas participantes. La figura 1 resume las categorías y las fuentes utilizadas para su explicación, que son descritas en detalle en este apartado. Cabe señalar que, siguiendo la metodología de caso 
múltiple, la información presentada en este artículo reporta generalidades que "calzan" con todos los casos. Las particularidades serán reportadas en otros manuscritos.

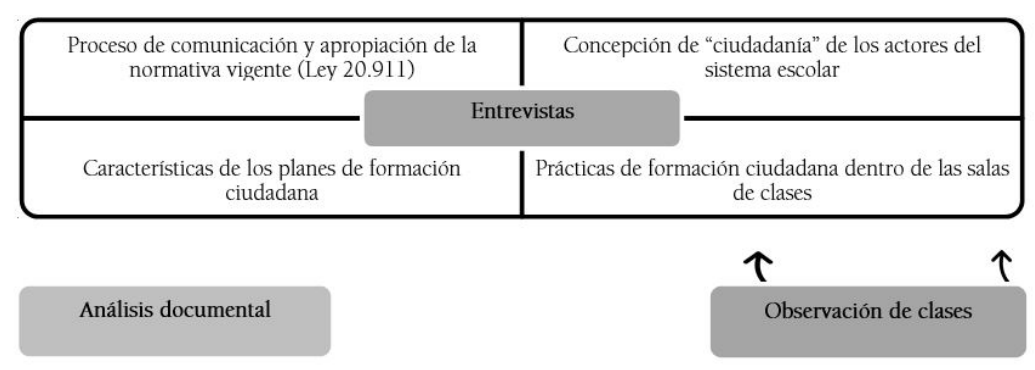

Figura $\mathrm{N}^{\circ} 1$

Ley № 20.911: procesos de comunicación y apropiación de la normativa vigente.

De acuerdo con las percepciones de los participantes, el proceso de apropiación de la normativa fue dificultoso, fundamentalmente porque, en algunas escuelas, solo consistió en asistir a una reunión convocada por la Secretaría Regional Ministerial de Educación, cuyo propósito era informar de las características de la Ley y los requerimientos del PFC. Dicha reunión informativa no fue sucedida por otras instancias de acompañamiento o seguimiento de la gestión de los colegios respecto del tema, por lo que cualquier tipo de dudas o inquietudes surgidas con posterioridad no fueron resueltas.

De esta manera, la sensación general, en cuanto a la información oficial recibida sobre la implementación del PFC, fue más bien de poca claridad e incertidumbre. Se observó mayor conocimiento de esta información por parte de directivos y encargados del PFC que por parte de los docentes entrevistados. Sin embargo, la vía de toma de conocimiento de la Ley 20.911 señalada por los distintos directivos no fue coincidente, lo cual deja en evidencia que la comunicación oficial a los establecimientos educacionales sobre la puesta en práctica de un PFC no fue óptima, si no más bien problemática.

Los directivos y encargados del PFC que tenían conocimiento de esta normativa admitieron que éste fue construido bajo la presión de ser evaluados por un ente fiscalizador externo, como la 
Superintendencia de Educación. Esta imposición se tradujo en PFC construidos en condiciones poco participativas, en las que una o dos personas estaban encargadas de su elaboración, diseñados de manera poco reflexiva, debido al escaso tiempo que tuvieron para su elaboración, lo cual fue considerado de manera transversal por los entrevistados como un problema.

El proceso de acompañamiento oficial del que dispusieron las escuelas para elaborar su PFC fue prácticamente inexistente. A algunos establecimientos se les ofreció apoyo externo desde instituciones de educación superior; sin embargo, ninguna de esas instancias llegó a concretarse, ya que los participantes señalaron que los dejaron solos en el proceso de elaboración del PFC, tal como evidencia el siguiente participante: "Vino un caballero de la universidad [...] y él estuvo con nosotros una mañana [...]. Supuestamente, nos iba a venir a ver, nos dio fecha. Hasta el día de hoy no ha llegado" (P7).

Un establecimiento pudo vivenciar el proceso de acompañamiento llevado a cabo por una institución de educación superior que se adjudicó la licitación del MINEDUC. Lamentablemente, esta instancia no fue provechosa, ya que, si bien eran convocados a reuniones para compartir experiencias, el acompañamiento al establecimiento y a los docentes fue escaso y esporádico. Esta situación generó en los participantes la percepción de que este acompañamiento era poco útil, lo que además provocó frustración frente a la poca ayuda recibida, expresada en la visión de uno de los entrevistados: "En lo personal, me parecía indigno, me parecía un fraude [...] volver a asistir, porque realmente ni siquiera ellos nos acompañaron" (P1).

Respecto de la relevancia de esta normativa para los establecimientos, los directivos y encargados del PFC sostienen que esta es una tarea más que cumplir entre las muchas que va requiriendo el MINEDUC: una imposición. El hecho de que el proceso de elaboración del PFC haya sido poco democrático, se tradujo en una apropiación e implementación poco efectiva, pues la sensación general fue que el PFC se construyó por cumplir con las disposiciones ministeriales, pero que realmente no constituyó una 
novedad o un aporte. Entonces, si bien los participantes declaran que la implementación de un PFC es una temática relevante y necesaria, por el modo en que fue impuesta desde el nivel central y por los escasos procesos de acompañamiento, la normativa carece de sentido para los establecimientos.

En las entrevistas, veintidós de los veintiséis docentes declararon desconocer el PFC de su escuela. Algunos participantes adjudican esta situación a responsabilidades personales, ya que, incluso, si se hubiera presentado el PFC en la escuela, por ejemplo, durante el Consejo de Profesores, ellos ya habían olvidado de qué se trataba, debido a la alta carga laboral que soportan y a la importancia que otorgan a otros ámbitos laborales, como el avance en la cobertura curricular. Esta responsabilidad personal es relacionada por otros profesores con la falta de proactividad que han tenido para apropiarse de las nuevas leyes, también en no haber solicitado el PFC a sus jefes directos, ni tampoco haberse ofrecido a ayudar en su elaboración. Incluso, un encargado del PFC de una escuela participante declaró, de manera autocrítica, que no se había dado el tiempo de informar sobre el PFC a los docentes que llegaron a integrar la planta docente del establecimiento.

Respecto de la comunicación efectiva sobre el PFC a los docentes, existen dos experiencias a destacar. En un establecimiento, desde 2017, un equipo de profesores de la escuela indagó sobre las acciones que realizaba la comunidad escolar con anterioridad a la promulgación de la Ley 20.911, y que podrían relacionarse con la formación ciudadana. De esta manera, los profesores entrevistados declararon estar en conocimiento de que algunos de sus colegas trabajaban en el PFC, e incluso comentaron que el marco teórico había sido difundido y socializado entre ellos (P17). Sin embargo, la difusión sobre los resultados finales de la elaboración del PFC aún no se realizaba cuando se recolectaron los datos para este estudio.

En otro establecimiento, la formación ciudadana había adquirido relevancia en la comunidad desde antes de la creación de la Ley 20.911. Años antes, la escuela creó una asignatura orientada a tratar temas contingentes y controversiales. Además, visibilizan la 
formación ciudadana desde una óptica participativa, por lo que los docentes declaran conocer las acciones del PFC y estar conscientes de la relevancia que tiene la intervención de todos los actores de la comunidad educativa para cumplir con las acciones planificadas, las que abarcan actividades como talleres, actos cívicos y debates para los estudiantes, e instancias recreativas para los profesores. De esta forma, los procesos de comunicación y apropiación de las acciones realizadas respecto de la formación ciudadana ha sido altamente efectiva en esta escuela.

\section{Características de los planes de formación ciudadana}

Los PFC analizados cumplen con las características que mandata la normativa vigente. Es decir, contienen acciones concretas derivadas de uno o varios objetivos presentes en la Ley, escogidos por los establecimientos, para luego describir en qué consiste la acción, el encargado, los recursos necesarios y los medios de verificación. El número de acciones presentes en los planes de los establecimientos participantes varía desde 3 hasta 21. Estas comprenden acciones concretas, que se desarrollaron tanto en 2018 como proyectadas para 2019. La tabla 4 resume el número de acciones contenidas en los PFC por cada escuela.

Al preguntar a los encargados de los PFC cómo surgen las acciones, ellos declaran que éstas se desarrollaban con anterioridad en sus establecimientos y que solo se consignaron en el plan. Indican que no han sido creadas especialmente para el PFC, lo que confirma la poca relevancia adjudicada a esta normativa. A su vez, como son acciones que ya existían, no necesariamente se vinculan con el Plan de Mejoramiento Educativo (PME) o con el Proyecto Educativo Institucional (PEI), como sugiere la Ley. Esto dificulta poder otorgar financiamiento a los PFC a través del PME, o que se produzca un alineamiento explícito con el tipo de persona que busca formar la institución a través del PEI. 
Tabla 4.

Número de acciones presentes en el Plan de Formación Ciudadana por escuela.

\begin{tabular}{cc}
\hline Escuela & $N^{\circ}$ de acciones \\
\hline Escuela 1 & 3 \\
\hline Escuela 2 & 9 \\
\hline Escuela 3 & 7 \\
\hline Escuela 4 & 18 \\
\hline Escuela 5 & 12 \\
\hline Escuela 6 & 7 \\
\hline Escuela 7 & 8 \\
\hline Escuela 8 & 21 \\
\hline
\end{tabular}

Fuente: elaboración propia.

Respecto de la naturaleza de las acciones, estas se relacionan principalmente con actividades extracurriculares realizadas dentro de la escuela, pero fuera de la sala de clases: por ejemplo, desarrollo de los centros de estudiantes y de padres, salidas pedagógicas, actos cívicos, ferias gastronómicas, efemérides, aniversarios del establecimiento, entre otros. Luego, se destacan numerosas acciones que se realizan en la comunidad, como visitas a la alcaldía u otra institución de gobierno, visitas a hogares de ancianos, limpieza y ornato de sectores aledaños a la escuela, participación en torneos escolares en otros establecimientos (como debates, ferias y actividades deportivas), entre otras. Estas son las que más se destacan por su potencial de formar ciudadanos, ya que permiten desarrollar sentido de pertenencia y requieren de una participación activa de los estudiantes para que puedan llevarse a cabo exitosamente. Finalmente, las acciones que se llevan a cabo dentro de la sala de clases están ligadas en su gran mayoría a la asignatura HGCS, y se basan en contenidos conceptuales y actitudinales contenidos en el Eje de Formación Ciudadana de las Bases Curriculares. De este modo, se incluyen actividades relacionadas con la valoración de espacios patrimoniales, la relevancia de los derechos humanos, la institucionalidad política, la participación electoral, entre otros. Lo anterior confirma la noción de que los docentes de HGCS son los principales encargados de promover la formación ciudadana en sus aulas, relegando a segundo plano el trabajo transversal que deberían realizar todos los docentes. 
156 ENTRE LA IMPOSICIÓN Y LA NECESIDAD: IMPLEMENTACIÓN DEL PLAN DE FORMACIÓN CIUDADANA EN ESCUELAS CHILENAS - C. G. Zúñiga, P. Ojeda, P. Neira, T. Cortés, M. J. Morel

Un aspecto a considerar sobre las acciones contenidas en los planes es que sus descripciones son ambiguas y generales, por lo que, mediante su lectura, no queda claro el espacio en que se realizan, la duración real de las actividades, la modalidad en que trabajarán los estudiantes o las habilidades que pretenden desarrollarse. Por lo tanto, el lector puede hacerse una idea de lo que tratan, pero no alcanza a comprender a cabalidad las características de su ejecución ni su alcance. Incluso, algunas acciones consignadas no necesariamente guardan relación con los objetivos de la Ley 20.911.

Concepción de ciudadanía de los actores del sistema escolar Cuando se les pregunta a los directores acerca de lo que significa ser "ciudadano", el concepto que más se menciona es el de "responsabilidad". Para ellos, ser ciudadano es tener derechos y responsabilidades. En cuanto a los derechos, se menciona el de libre expresión, a elegir y ser elegido, y a tomar decisiones; en cuanto a los deberes, mencionan que es necesario estar informados de lo que ocurre y opinar con argumentos. Es importante también, para los directores, modelar lo que entienden por ciudadanía; en otras palabras, dar el ejemplo en cuanto al respeto y el trato justo, o reforzar valores de un buen ciudadano.

En el caso de los demás docentes, la ciudadanía tiene relación con pertenecer a una comunidad, con la obtención de derechos y deberes, y se identifica el voto como un deber ciudadano. Se da especial relevancia a la idea de participar en el lugar al que se pertenece, acción puede darse a partir del voto o en otras instancias de toma de decisiones, como la junta de vecinos. Es interesante destacar que en los profesores surge la idea de aportar a la sociedad ejerciendo la docencia, valorando su práctica profesional como parte de la concepción práctica del ser ciudadano, lo que es retratado por uno de los participantes: "Mi rol como ciudadano es aportar a la sociedad a través de la educación" (P38).

En los estudiantes emerge la idea de que ser ciudadano es formar parte de una comunidad, participar activamente y cuidar esta comunidad, a las personas y al entorno. Una de las principales definiciones de "ciudadano", según los alumnos, es tener voz y voto. 
El sufragio lo asocian con hacer un bien al país, o con una atribución que les permite criticar y opinar. Sin embargo, en su mayoría, los estudiantes participantes no se consideran ciudadanos porque son menores de 18 años.

Para los estudiantes, el respeto también aparece como un valor esencial a la hora de definir al ciudadano. Según ellos, un buen ciudadano debe respetar la opinión del otro, aunque piense distinto; debe respetar el espacio del otro, las decisiones de la gente y, por tanto, a las autoridades que se eligen. En este sentido, los participantes enfatizan la necesidad de que la pertenencia y la participación se den en un ambiente de respeto. Es así como el respeto aparece como un derecho y, a la vez, como un deber.

La distancia entre las responsabilidades de la escuela y de los directores en la promoción de la ciudadanía es difusa, especialmente en la idea de generar espacios de participación y de expresión, ya que es vista como una obligación de las escuelas, pero los directores declaran tener el deber de llevarla a cabo y de disponer recursos.

También aparece como una obligación para las escuelas mantener a los alumnos informados respecto de sus derechos y deberes, formar a niños y apoderados acerca de la ciudadanía y generar conciencia sobre sus responsabilidades. Lo anterior es corroborado por parte de los estudiantes. Los docentes también otorgan gran parte de la responsabilidad a las escuelas, señalando que muchas veces la escuela es el único espacio donde los estudiantes pueden formarse en valores ciudadanos, que permiten desenvolverse en la sociedad.

Al definir el concepto de "ciudadanía" surgen similitudes entre los diversos participantes, asimilando la noción de "ciudadano" al de pertenencia a una comunidad. Sin embargo, cuando se les pregunta a los estudiantes cómo las escuelas les enseñan a ser ciudadanos, hay alumnos que perciben que la escuela no cumple ese rol. Al respecto, un participante indica que en las escuelas no se les enseñan esos temas, ya que el colegio "es como para estudiar no más, para salir adelante, pero no es para hacer política así. No tiene nada que ver con eso" (Esc3, Est2). 
Prácticas de formación ciudadana dentro de las salas de clases

Una de las prácticas de promoción de la formación ciudadana frecuentemente observada en las salas de clases fue el clima de aula caracterizado por el respeto y el trato afable entre los distintos actores. Esto quedó expuesto en que los profesores trataban por su nombre a los estudiantes, se demostraba proximidad física y sonrisas entre los protagonistas, incluso risas o bromas entre ellos. Todos estos elementos configuraban un lugar seguro para que los estudiantes pudieran participar.

De esta manera, las aulas observadas se caracterizan por un clima abierto a la participación en la sala de clases, en la cual los estudiantes pueden responder a preguntas abiertas y cerradas formuladas por los docentes, dirigidas a algunos de manera personal y otras orientadas al curso completo, permitiendo la participación de cualquier estudiante. Este espacio fomentado por los docentes es aprovechado por los alumnos, verificando un alto protagonismo de ellos en clases. Además, se alternan la palabra entre hombres y mujeres, sin evidenciar sesgo de género en las clases observadas.

No obstante, la mayoría de las veces esta participación está limitada a responder preguntas relacionadas con la repetición del contenido, centradas en la memorización, y con habilidades cognitivas simples, como reconocer, identificar o recordar información de clases anteriores. Tal participación no implica el desarrollo de habilidades más complejas, como inferir, concluir, crear o hipotetizar. En síntesis, el espacio que incentiva la participación de los estudiantes — donde no se condenan las equivocaciones, sino que se utilizan los errores como instancias de aprendizajes - se utiliza en preguntas poco desafiantes y significativas para los alumnos. No obstante, un aula abierta a la participación se configura como un muy buen primer paso para generar una ciudadanía crítica en los niños y jóvenes, lo que incluye relacionar los contenidos curriculares con noticias contingentes o controversiales, con otras asignaturas y con el contexto de los estudiantes.

Existen casos excepcionales que dan cuenta de un espacio que fomenta el pensamiento crítico de los estudiantes, como son las clases 
de dos profesores de ciencias de distintos colegios. Estos profesores buscaban en sus clases que los estudiantes hipotetizaran, infirieran y conectaran los contenidos con su realidad a través del pensamiento científico y la experimentación. Esta modalidad de aprendizaje fomenta que los estudiantes "aprendan haciendo" e investiguen, siempre desde la vinculación con su realidad, para generar interés por su aprendizaje, por ejemplo, al pedirles que den una opinión fundamentada sobre un tema en particular.

La observación de clases deja como evidencia una baja inclusión de actividades de aprendizaje activo con alto protagonismo de los estudiantes, y poca diversidad de actividades de aprendizaje, lo que se manifiesta como una de las debilidades observadas, ya que incluir más de estas prácticas democratizaría el conocimiento en el aula.

Finalmente, en las entrevistas realizadas a profesores de HGCS se evidencia que en su mayoría perciben como sinónimos los términos "formación ciudadana" y "educación cívica". Esto a pesar de que las orientaciones curriculares del MINEDUC, en los últimos años, las han diferenciado claramente. Como resultado, las prácticas de estos docentes se alejan de la enseñanza de una ciudadanía participativa y se orientan hacia la memorización de conceptos y el conocimiento institucional y político del Estado.

\section{Discusión}

Las escuelas deben definir colaborativamente su propia concepción de ciudadanía

Las orientaciones emanadas desde el MINEDUC señalan que en la formulación del PFC debiera participar toda la comunidad educativa mediante a un proceso de reflexión comunitaria (MINEDUC, 2016a), pero en la práctica se observa que la elaboración recae principalmente en el docente encargado. Desde la génesis del PFC no existe una discusión o reflexión sobre el tipo de ciudadano que quiere formar la escuela, de acuerdo con el contexto y las particularidades de cada comunidad educativa. Por lo tanto, la participación de los diversos 
actores queda reducida al conjunto de acciones que el plan estipula que debieran realizar, siendo más una imposición que una visión y meta en conjunto. Esta lógica de creación poco participativa coincide con los hallazgos del PNUD (2018), en los cuales no se involucra a la comunidad educativa en la creación del PFC. Esta forma de implementación de las políticas educativas en las escuelas (Walshaw \& Anthony, 2007) reduce sus posibilidades como espacio democrático.

El MINEDUC propone que este plan se articule con el Proyecto Educativo Institucional (PEI) y con el Plan de Mejoramiento Educativo (PME) de la escuela (MINEDUC, 2016a). Sin embargo, no se aprecia un conjunto de actividades articuladas, sino, más bien, pareciera ser que la implementación del PFC viene siendo otra carga extra a los planes y programas que ya existen en las escuelas. Todo lo anterior genera que no exista una visión común respecto de la ciudadanía y del rol del ciudadano, y, por tanto, tampoco existe una participación coordinada de los distintos actores. Esto no solo dificulta la implementación del PFC, sino que además afecta el fomento de la ciudadanía en la escuela.

Por otro lado, no existe por parte de los participantes una diferencia conceptual entre "ciudadanía" y "ciudadano". Más bien, conviven las ideas de la pertenencia a un espacio y de la participación electoral como práctica ciudadana. Además, es común que se hable de la existencia de derechos y deberes, sin necesariamente señalar cuáles son estos. En este sentido, la aproximación al concepto "ciudadanía" por parte de directivos y docentes parece ser intuitiva, relacionada con su experiencia personal y su visión del rol que debería tener la educación.

Con esto no se pretende que debiera existir una visión única de "ciudadanía". Se entiende que el concepto es polisémico y con distintas interpretaciones, pero para promover la formación ciudadana en las escuelas debe existir una concepción de "ciudadanía" que surja de común acuerdo en la comunidad escolar, ya que solo así ésta podrá actuar coordinadamente para fomentarla en la escuela. En Chile la política es generada desde arriba y la escuela debe implementarla; 
por tanto, los actores educativos no son partícipes en la creación de las diversas normativas, sino que se les imponen (Olson, 2002). Con mayor razón, la creación de esta conceptualización debiera estar guiada y asegurada durante la implementación de esta política pública.

A esto se suma el escaso acompañamiento a las escuelas durante la implementación de la política. Siete de los ocho establecimientos participantes informaron no haber recibido acompañamiento ni apoyo en la creación e implementación del plan, y la única institución que informa haberlo tenido no quedó satisfecha con la asesoría. En este sentido, cabe preguntarse sobre la utilidad de que, desde el nivel central, emanen prescripciones que deben ser acatadas por los establecimientos, sin proveer apoyos concretos y suficientes que permitan generar cambios reales en las escuelas.

La necesidad de generar prácticas de aula para la formación de ciudadanos

A pesar de que el aprendizaje para la ciudadanía se considera actualmente un objetivo transversal en el currículo escolar - en el que se espera que los estudiantes, mediante las distintas asignaturas en la escuela, estén preparados para ejercer una ciudadanía activa-, los profesores adjudican la responsabilidad de este eje curricular al profesor que imparte la asignatura de HGCS. Esta realidad se condice con los hallazgos de Jara, Sánchez y Cox (2019), que demuestran que los profesores de HGCS concentran las responsabilidades de formación ciudadana en los contenidos de la asignatura, y con el análisis realizado por la Agencia de Calidad de la Educación (2016a), que expone que los contenidos de formación ciudadana son relacionados con la historia, incluso relegando a un menor espacio el resto de las ciencias sociales.

Sin embargo, los profesores de HGCS que participaron de este estudio reafirmaron que la formación ciudadana es un objetivo transversal del currículo escolar y, por lo tanto, responsabilidad de los docentes de todos los niveles y todas las asignaturas. Los profesores de esta asignatura entregan poco énfasis a la formación ciudadana, debido al apremio que tienen de cumplir con la cobertura curricular, 
162 ENTRE LA IMPOSICIÓN Y LA NECESIDAD: IMPLEMENTACIÓN DEL PLAN DE FORMACIÓN CIUDADANA EN ESCUELAS CHILENAS - C. G. Zúñiga, P. Ojeda, P. Neira, T. Cortés, M. J. Morel

lo que no les da espacio para profundizar en estos contenidos. La visión de la formación ciudadana como un pilar secundario del currículo se reafirma por la apropiación incorrecta de la definición de "formación ciudadana" —limitada a una visión anacrónica ligada a la educación cívica—, alejada de las orientaciones ministeriales, que la promueven en cambio como un aprendizaje basado en competencias, vinculado a las actitudes, habilidades y conocimientos ciudadanos.

Esto queda reflejado, aún más, en las prácticas de aula de los profesores de HGCS, que promueven un espacio de participación ligado a la repetición y memorización de contenidos relativos a la institucionalidad política y al funcionamiento del Estado. Estos hallazgos coinciden con investigaciones que dan cuenta de que el foco de los docentes en el aula está puesto en sus propias intervenciones y en modelos tradicionales de enseñanza, en los cuales las estrategias pedagógicas utilizadas no dan espacio a interacciones democráticas para que los estudiantes construyan conocimiento (Cavieres y Muñoz, 2015; Bonhomme et al., 2015). A su vez, concuerdan con que, si bien existe expresión de ideas por parte de los estudiantes, estas se relacionan con temáticas curriculares, guiadas desde el docente y sin profundizar en asuntos de contingencia nacional o internacional (Agencia de Calidad de la Educación, 2016b). Esta situación distancia las prácticas de los profesores de HGCS de la promoción de ciudadanos críticos e informados. Ello tiene mayor relevancia en el actual contexto nacional, en el que, desde 2020, los docentes de esta asignatura deberán impartir la asignatura Formación Ciudadana para los niveles de $3^{\circ}$ y $4^{\circ}$ medio. Si bien al momento de informar los resultados de esta investigación no se encontraban disponibles los programas de estudio de esta asignatura, su enfoque se vincula con la formación ciudadana y el desarrollo de habilidades necesarias para formar ciudadanos participativos (Westheimer \& Kahne, 2004), que sean capaces de desenvolverse activamente en sus contextos. Entonces, si estos docentes en sus clases de HGCS continúan enfatizando solo el elemento cívico, es de esperar que repliquen esta práctica en la recién creada asignatura, lamentablemente. De este modo, es imperativo que, desde el MINEDUC y las carreras de pedagogía, se desarrollen instancias de formación continua, que entreguen apoyo a estos docentes con el fin de superar el enfoque de la educación cívica. 


\section{Conclusiones}

Es necesario fomentar la promoción transversal de la formación ciudadana en la escuela, la que debe estar a cargo de los docentes de todos los niveles y de todas las asignaturas. El gran problema de la transversalidad, ya evidenciado en su intento fallido de promoverla desde la Reforma Educacional de los 90, es que, al ser responsabilidad de todos, cae en terreno de nadie. Inmersos en una cultura de evaluaciones de altas consecuencias, este problema se acrecienta, al no ser un tema de estudio evaluado por las mediciones estandarizadas implementadas en el país, como el SIMCE o la PSU. Si bien en el SIMCE se incluye un cuestionario sobre esta materia, este no se incluye en el puntaje que se reporta a los establecimientos. Una posible solución puede encontrarse en la formación inicial docente, de modo de incluir la formación ciudadana como asignatura de todas las carreras de pedagogía. De este modo, los futuros docentes podrían comprender cuál es su responsabilidad en la formación de ciudadanos.

Al respecto, la mayoría de los participantes comparte la noción de que la labor de promover la formación ciudadana recae fundamentalmente en los docentes de HGCS, ignorando que, en diversas situaciones, están promoviendo ciudadanía en sus asignaturas de manera efectiva, aunque no sea de manera intencionada, mediante prácticas como un clima de aula favorable al aprendizaje, vinculación con los estudiantes, lenguaje cercano y proximidad física. Incluso, en algunos casos, la promoción de ciudadanía es mayor en ellos que en los docentes de HGCS, por lo que hace falta relevar y divulgar estas prácticas que ya realizan los docentes en el aula, con el fin de que puedan promocionarlas de manera consciente e intencionada (Zúñiga et al., 2020). Entre los docentes participantes destacaban las prácticas de algunos profesores de Ciencias Naturales, debido a que brindaban a sus estudiantes la oportunidad de tener experiencias de aprendizaje activo a partir de la aplicación de metodologías de indagación, propias de las disciplinas de origen.

Otra alternativa se encuentra en la promoción del rol social del docente como actor de cambio social. Esto podría permitir superar los 
problemas tanto de la visibilización de las prácticas no intencionadas como de la falta de transversalidad de la formación ciudadana, al empoderar a los docentes en su rol de promotores de ciudadanía. De todas maneras, para superar estos problemas, es clave el rol de las políticas públicas a futuro, para fomentar la enseñanza de la ciudadanía como un eje clave dentro de la Formación Inicial Docente.

A raíz de lo expuesto, es de suma importancia generar recomendaciones respecto de la implementación de la política pública, específicamente en la creación y puesta en marcha del $\mathrm{PFC}$ en las escuelas. Es importante que este plan sea construido de manera democrática y participativa, que se apoye a las escuelas en este proceso y que no sea solo una fiscalización enfocada en la existencia del PFC. El acompañamiento a las escuelas es parte de la ley y la evidencia de esta investigación ha demostrado que esto no se cumple en la práctica.

En relación con las prácticas de aula que promueven la formación de ciudadanos, se recomienda integrar esta temática en la formación inicial y continua de los docentes, de manera de eliminar la carga que recae en la asignatura de HGCS, e incluirla explícitamente en todas las asignaturas y niveles. Lo anterior debe estar acompañado de un sistema de divulgación de las prácticas de formación ciudadana que hoy realizan los docentes de distintas asignaturas en Chile, y que muchas veces no las relacionan con esta temática. Estas recomendaciones podrían mejorar la puesta en marcha de una política pública que fue generada fuera de las escuelas, y cuyos objetivos no han logrado impactar en el quehacer de docentes y directivos.

\section{Referencias}

Abs, H. (2009). Learning and living in democracy. Introducing quality assurance of education for democratic citizenship in schools. Comparative study of 10 countries. Paris: Council of Europe Publishing.

Agencia de Calidad de la Educación. (2016b). Los indicadores de desarrollo personal y social en los establecimientos educacionales chilenos: una primera mirada. Recuperado de: http://archivos.agenciaeducacion. 
cl/estudios/Estudio_Indicadores_desarrollo_personal_social_en_ establecimientos_chilenos.pdf

Agencia de Calidad de la Educación. (2016a). Formación Ciudadana en el sistema escolar chileno:una mirada a las prácticas actuales y recomendaciones de mejora. Santiago de Chile: Agencia de la Calidad de la Educación.

Agencia de Calidad de la Educación. (2018). Informe nacional ICCS 2016. Recuperado de: https://www.iea.nl/sites/default/files/2019-07/ ICCS_2016_National_Report_CHL.pdf

Alivernini, F. \& Manganelli, S. (2011). Is there a relationship between openness in classroom discussion and students' knowledge in civic and citizenship education? Procedia-Social and Behavioral Sciences, 15, 3441-3445.

Avery, P., Levy, S. \& Simmons, A. (2013). Deliberating controversial public issues as part of civic education. The Social Studies, 104(3), 105-14.

Bonhomme, M., Cox, C., Tham, M. y Lira, R. (2015). La Educación Ciudadana escolar de Chile 'en acto': prácticas docentes y expectativas de participación política de los estudiantes. En C. Cox \& J. Castillo (eds.), Aprendizaje de la ciudadanía. Contextos, experiencias y resultados (pp. 373-425). Santiago de Chile: Ediciones Universidad Católica de Chile.

Brint, S. (2006). School and societies. Stanford: Stanford University Press.

Cárdenas, M. (2017). Developing pedagogical and democratic citizenship competencies. "Learning by Participating" Program. In B. García, A. Sandoval, E. Treviño, S. Diazgranados \& M. Pérez (eds). Civics and Citizenship: Theoretical Models and Experiences in Latin America (pp. 207-239). Rotterdam: Sense Publishers.

Cavieres, E., y Muñoz, J. (2015). Pensamiento de los profesores de historia acerca de la ciudadanía y la inclusión de las movilizaciones estudiantiles en su enseñanza. Estudios Pedagógicos, 41(2), 55-70.

Charmaz, K. (2006). Constructing grounded theory: A practical guide through qualitative analysis. London: Sage.

Corbin, J. \& Strauss, A. (2008). Basics of qualitative research: Techniques and procedures for developing grounded theory. Los Ángeles: Sage.

Cox, C. y Castillo, J. (2015). Aprendizaje de la ciudadanía. contextos, experiencias y resultados. Santiago de Chile: Ediciones UC.

Darling-Hammond, L., Amrein-Beardsley, A., Haertel, E. \& Rothstein, J. (2012). Evaluating teacher evaluation. Phi Delta Kappan, 93(6), 8-15. 
166 ENTRE LA IMPOSICIÓN Y LA NECESIDAD: IMPLEMENTACIÓN DEL PLAN DE FORMACIÓN CIUDADANA EN ESCUELAS CHILENAS - C. G. Zúñiga, P. Ojeda, P. Neira, T. Cortés, M. J. Morel

Engel, E. (2015). Consejo asesor presidencial sobre los conflictos de interés, el tráfico de influencias y la corrupción, Informe Final. Recuperado de: http://www.primerapiedra.cl/documentos/informeconsejo.pdf

Flick, U. (2009). An introduction to qualitative research. London: Sage.

Flick, U. (2014). La gestión de la calidad en investigación cualitativa. Barcelona: Morata.

Folgueiras, P., Massot, I. y Sabariego, M. (2008). La ciudadanía activa e intercultural en alumnado de la ESO. Revista Electrónica Interuniversitaria de Formación Del Profesorado, 11(3), 10-22.

Geboers, E., Geijsel, F., Admiraal, W. \& ten Dam, G. (2013). Review of the effects of citizenship education. Educational Research Review, 9, 158-173.

Gubrium, J. \& Holstein, J. (2005). Interpretive practice and social action. In N. Denzin \& Y. Lincoln (eds.) The SAGE Handbook of Qualitative Research (pp. 483-505). London: Sage.

Hahn, C. L. (2016), 'Pedagogy in citizenship education research: A comparative perspective', Citizenship Teaching \& Learning, 11(2), 121137. DOI: 10.1386/ctl.11.2.121_1

Hammersley, M. \& Atkinson, P. (1995). Ethnography: Principles in practice. London: Routledge.

Haste, H. (2004). Constructing the citizen. Political Psychology, 25, 413-439.

Haste, H., Bermúdez, A. \& Carretero, M. (2017). Culture and Civic competence; Widening the Scope of the Civic Domain. In B. García, A. Sandoval, E. Treviño, S. Diazgranados, S. \& M. Pérez (eds). Civics and Citizenship: Theoretical Models and Experiences in Latin America (pp. 3-16). Rotterdam: Sense Publishers.

Henríquez, R. y Mardones, R. (2016). Educación y ciudadanía. En I. Sánchez (ed.), Ideas en educación/ Reflexiones y propuestas desde la UC (pp. 572 600). Santiago de Chile: Ediciones UC. DOI: https://doi.org/10.13140/ RG.2.1.3720.8089

Hess, D. \& McAvoy, P (2015). The Political Classroom: Evidence and Ethics in Democratic Education. New York: Routledge.

Jara, C., Sánchez, M. y Cox, C. (2019) Liderazgo educativo y formación ciudadana: visiones y prácticas de los actores. Calidad en la Educación, $51,350-381$.

Kahne, J., Hodgin, E. \& Eidman-Aadahl, E. (2016). Redesigning civic education for the digital age: Participatory politics and the pursuit of democratic engagement. Theory \& Research in Social Education, 44(1), 1-35. 
Keating-Chetwynd, S. (2009). How all teachers can support citizenship and human rights education: a framework for the development of competences. Belgium: Council of Europe Publishing. Recuperado de: https:// rm.coe.int/CoERMPublicCommonSearchServices/DisplayDCTMCon tent?documentId=09000016802f726a

Knowles, R. \& McCafferty-Wright, J. (2015). Connecting an open classroom climate to social movement citizenship: A study of 8th graders in Europe using IEA ICCS data. The Journal of Social Studies Research, 9(4), 255-269. DOI: https://doi.org/10.1016/j.jssr.2015.03.002.

Litchman, M. (2010). Qualitative research in education: A user's guide. Thousand Oaks, CA: Sage.

Luengo, P. \& Jiménez, G. (2017). Good practices on civic engagement in Chile and the role of promoting prosocial behaviors in school settings. In B. García, A. Sandoval, E. Treviño, S. Diazgranados \& M. Pérez (eds). Civics and Citizenship: Theoretical Models and Experiences in Latin America (pp. 240-254). Rotterdam: Sense Publishers.

Martínez, F., Taut, S. \& Schaaf, K. (2016). Classroom observation for evaluating and improving teaching: An international perspective. Studies in Educational Evaluation, 49, 15-29. DOI: https://doi. org/10.1016/j.stueduc.2016.03.002

Martínez, M., Silva, C. y Hernández, A. C. (2010). ¿En qué ciudadanía creen los jóvenes? Creencias, aspiraciones de ciudadanía y motivaciones para la participación sociopolítica. Psykhe, 19(2), 25-37.

McAvoy, P. \& Hess, D. (2013). Classroom deliberation in an era of political polarization. Curriculum Inquiry, 43(1), 14-47.

Merriam, S. B. (2009). Qualitative research: A guide to design and implementation. San Francisco: Jossey-Bass.

Ministerio de Educación [MINEDUC]. (1999). Curriculum de la educación media. Objetivos fundamentales y contenidos mínimos obligatorios. Santiago de Chile: MINEDUC.

Ministerio de Educación [MINEDUC]. (2009). Objetivos fundamentales y contenidos minimos obligatorios de la educación básica y media: Actualización 2009. Santiago de Chile: Ministerio de Educación.

Ministerio de Educación [MINEDUC]. (2013). Bases curriculares Educación Básica. Santiago de Chile: Ministerio de Educación.

Ministerio de Educación [MINEDUC]. (2015). Bases curriculares $7^{\circ}$ básico a $2^{\circ}$ medio. Santiago de Chile: Ministerio de Educación. 
168 ENTRE LA IMPOSICIÓN Y LA NECESIDAD: IMPLEMENTACIÓN DEL PLAN DE FORMACIÓN CIUDADANA EN ESCUELAS CHILENAS - C. G. Zúñiga, P. Ojeda, P. Neira, T. Cortés, M. J. Morel

Ministerio de Educación [MINEDUC]. (2016a). Orientaciones para la elaboración del Plan de Formación Ciudadana. Santiago de Chile: Ministerio de Educación.

Ministerio de Educación [MINEDUC]. (2016b). Orientaciones curriculares para el desarrollo del Plan de Formación Ciudadana. Santiago de Chile: Ministerio de Educación

Muñoz, C., Martínez, R., y Muñoz, C. (2016). Percepciones del estudiantado sobre la política, los partidos políticos y las personas dedicadas a la política al finalizar la educación secundaria en Chile. Revista Electrónica Educare, 20(1), 1-16. DOI: http://dx.doi.org/10.15359/ree.20-1.17

Olson, J. (2002). Systemic change/teacher tradition: Legends of reform continue. Journal of Curriculum Studies, 34(2), 129-137. DOI: https:// doi.org/10.1080/00220270110085697

Programa de las Naciones Unidas para el Desarrollo [PNUD]. (2018). Estudio sobre la puesta en marcha del Plan de Formación Ciudadana. Santiago de Chile: Programa de las Naciones Unidas para el Desarrollo.

Puig, M. y Morales, J. (2015). La formación de ciudadanos: Conceptualización y desarrollo de la competencia social y cívica. Educación XX1, 18(1), 259-282. DOI: 10.5944/ educXX1.18.1.12332

República de Chile. (2016, abril 2). Ley N²0.911. Crea el plan de formación ciudadana para los establecimientos educacionales reconocidos por el Estado. Diario Oficial, Santiago de Chile.

Reyes, L., Campos, J., Osandón, L. y Muñoz, C. (2013). El profesorado y su rol en la formación de los nuevos ciudadanos. Estudios Pedagógicos, 39(1), 217-237.

Ross, W. y Vinson, K. (2012). La educación para una ciudadanía peligrosa. Enseñanza de las ciencias sociales, 11, 73-86.

Schulz, W., Ainley, J., Fraillon, J., Kerr, D. \& Losito, B. (2010). ICCS 2009 International Report: Civic knowledge, attitudes and engagement among lower secondary school students in thirty-eight countries. Amsterdam: International Association for the Evaluation of Educational Achievement, IEA.

Shulman, L. (1986). Those Who Understand: Knowledge Growth in Teaching. Educational Researcher, 15(2), 4-14.

Torney-Purta, J. (2004). Adolescents' political socialization in changing contexts: An international study in the spirit of Nevitt Sanford. Political Psychology, 25, 465-478. 
Treviño, E., Villalobos, C., Béjares, C. y Naranjo, E. (2019). Forms of Youth Political Participation and Educational System: The Role of the School for 8th Grade Students in Chile. YOUNG, 27(3), 279-303. DOI: https://doi.org/10.1177/1103308818787691

Walshaw, M. \& Anthony, G. (2007). Policy implementation: Integrating the personal and the social. Mathematics Teacher Education and Development, 8, 5-22. Recuperado de http://www.merga.net.au/ documents/MTED_8_Walshaw.pdf

Westheimer, J. \& Kahne, J. (2004). What kind of citizen? The politics of educating for democracy. American Educational Research Journal, 41(2), 237-269.

Zúñiga, C., Cárdenas, P., Martínez, R. \& Valledor, L. (2020) Teachers' classroom practices for citizenship education: Experiences of teachers rated as outstanding. Citizenship, Social and Economics Education, 19, 1. DOI: https://doi.org/10.1177/2047173419887972

Recibido: 15/11/2019

Aceptado: 07/04/2019 